\title{
Análisis cualitativo del nivel de satisfacción de la educación virtual en estudiantes universitarios en tiempos de pandemia
}

\author{
Israel Barrutia Barreto1, Juan José Danielli Rocca², Renzo Seminario \\ Córdova $^{1}$ y Paola Monzón Narciso ${ }^{1}$
}

\author{
${ }^{1}$ Innova Scientific SAC, Perú | ibarrutia2021@gmail.com; rseminario@alumni.unav.es; \\ paolalmonzonn@gmail.com | https://orcid.org/0000-0002-5728-0651; https://orcid.org/0000- \\ 0001-6992-5990; https://orcid.org/0000-0002-6100-7885 \\ ${ }^{2}$ Departamento Académico de Psicología Universidad Nacional Mayor de San Marcos, Perú \\ | daniellijuanjo@hotmail.com| https://orcid.org/0000-0001-5453-1956
}

\begin{abstract}
Resumen: Introducción. Ante el actual contexto sanitario mundial, las personas han cambiado sus estilos de vida acorde a los nuevos lineamientos de salud. En ese sentido, el sector educativo es uno de los que más cambios han realizado, puesto que ha habido una abrupta transición de la educación presencial a la educación virtual. Sin embargo, este cambio ha impactado en diferentes formas a los estudiantes universitarios. Objetivos. Describir el nivel de satisfacción respecto al nuevo sistema de educación virtual por estudiantes universitarios de Lima, en época de pandemia Covid-19. Metodología. La información se recolectó en base a encuestas online distribuidas mediante correo electrónico y redes sociales, las cuales fueron respondidas por un total de 10 estudiantes. La encuesta estuvo compuesta por 7 preguntas enfocadas en el grado de satisfacción de los estudiantes por las facilidades en el uso de herramientas informáticas brindadas por la universidad y el estilo de enseñanza de los docentes para transmitir los conocimientos. Resultados y discusiones. Los estudiantes mostraron un nivel de satisfacción moderado respecto a las clases recibidas en la modalidad online. Esto debido a la falta de alfabetización en recursos informáticos, especialmente, en los docentes. Asimismo, muchos de ellos indicaron que aún no se acostumbran a esta nueva modalidad online, puesto que sienten una mayor carga de trabajos. Es por ello que, es de suma importancia continuar mejorando los recursos informáticos ofrecidos por la institución y que los docentes tengan capacitaciones para ofrecer una enseñanza más didáctica y con ello, lograr captar la atención de los estudiantes. Conclusiones. Los resultados indican que, para los estudiantes universitarios la adaptación a la educación virtual se produjo progresivamente, desde inicios categorizados como "difíciles" hacia la "adaptación" al nuevo esquema, al que aún perciben deficiente en relación a la modalidad presencial.
\end{abstract}

Palabras clave: Satisfacción; Universidad; Educación Virtual; Alfabetización Informática.

Qualitative Analysis of the Level of Satisfaction with Virtual Education in University Students in Times of Pandemic

Abstract: Introduction. Given the current global health context, people have changed their lifestyles according to the new health guidelines. In this sense, the education sector is one of the sectors that has made the most changes, since there has been an abrupt transition from face-to-face education to virtual education. However, this change has impacted college students in different ways. Goals. To describe the level of satisfaction with the new virtual education system by university students in Lima, during the Covid-19 pandemic. Methodology. The information was collected based on online surveys distributed via email and social networks, which were answered by a total of 10 students. The survey consisted of 8 questions focused on the degree of student satisfaction with the facilities in the use of computer tools provided by the university and the teaching style of teachers to transmit knowledge. Results and discussions. The students showed a moderate level of satisfaction with the classes received in the online mode. This is due to the lack of literacy in computer resources, especially in teachers. Likewise, many of them indicated that they are still not used to this new online modality, since they feel a greater workload. That is why, it is of utmost importance to continue improving the computer resources offered by the institution and for teachers to have training to offer a more didactic teaching and thus, to attract the attention of students. Conclusions. The results indicate that, for university students, adaptation to virtual education occurred progressively, from beginnings categorized as "difficult" towards "adaptation" to the new scheme, which they still perceive as deficient in relation to the face-toface modality.

Keywords: Satisfaction; University; Virtual Education; Computer Literacy. 


\section{Introducción}

La actual pandemia del Covid-19 ha impactado en todo el mundo y obligando a la sociedad a cambiar costumbres y formas de vivir. Entre los diferentes sectores que ha impacto esta situación se encuentran salud, economía y, principalmente, el educativo (Cáceres, Jiménez \& Martín-sánchez, 2020). El sector educativo, ha tenido que innovar múltiples formas de adaptación, puesto que esta situación sanitaria tiene una alta probabilidad de extenderse por largo tiempo. Es así como la educación presencial deja de ser la principal forma de aprender para dar lugar a la educación virtual (Martínez-Garcés \& GarcésFuenmayor, 2020). En otras palabras, la educación virtual debe garantizar una alta calidad educativa.

En ese sentido, la educación virtual se ha ido convirtiendo en el pilar de la nueva forma de aprendizaje desde el inicio de la pandemia. Según Trujillo Marulanda \& Bustamante (2009), la educación virtual ofrece una nueva perspectiva, tanto a estudiantes como docentes, para el uso de diferentes técnicas y herramientas en el proceso enseñanzaaprendizaje. Dentro de esta modalidad pedagógica el Aula Invertida se ha posicionado ubicando en los niveles inferiores de la taxonomía de Blohm el comprender y memorizar. ascendiendo las categorías aplicar, analizar, evaluar y ubicando en la cúspide la categoria crear que comprende la acción innovadora del proceso (Hernández y Tecpan, 2017; Zainuddin \& Halili, 2016). El desplazamiento de las categorías en la taxonomía de Blohm, se manifiesta durante el ejercicio dela educación virtual y colaborativa, donde el estudiante adquiere rol activo en la interacción pedagógica, desarrollando competencias nuevas con vocación innovadora y orientadas hacia la generación de producto. En ese orden, es fundamental incorporar la educación virtual dentro de la malla curricular de los estudiantes, especialmente, los de educación superior (González, 2019; Durán, Estay-Niculcar \& Álvarez, 2015).

La pandemia precipitó la adecuación a la educación virtual forzando la adaptación pedagógica a todos los niveles educativos. A nivel universitario, la sociedad ha colocado una obligación onerosa sobre las instituciones y docentes orientado hacia la implementación de estrategias didácticas multimedia (Cáceres, 2020). En consecuencia, la evaluación continua, referida a la calidad de la enseñanza y la satisfacción de los estudiantes respecto a la educación virtual es imperiosa (De la Fuente, Marzo \& Reyes, 2010; Olivera, 2020).

Esta investigación tuvo como objetivo describir el nivel de satisfacción respecto al nuevo sistema de educación virtual por estudiantes universitarios en Lima, en época de pandemia Covid-19.

\section{Metodología}

\subsection{Tipo y Diseño De La Investigación}

El presente estudio fue de diseño no experimental, cualitativo y descriptivo, como estrategias efectivas para la investigación educativa (Hernández, Fernández \& Baptista, 2014; Manterola, Quiroz, Salazar \& García, 2019). El enfoque fue fenomenológico, debido a que el estudio se enfoca en la recopilación de experiencias individuales subjetivas de los participantes (estudiantes universitarios y recién egresados) en relación con un fenómeno colectivo -implementación de la modalidad educativa virtual durante la pandemia) (Relinque, Moral \& González, 2013; Salgado, 2007). 


\subsection{Participantes}

La selección de participantes en la presente investigación no obedeció los criterios probabilísticos, esto debido a que, en los estudios cualitativos la determinación de la muestra se realiza de manera intencional (Hernández et al., 2014), de manera que el investigador se posicione en una situación que mejor le permita recoger la información relevante para responder a la pregunta de investigación planteada (Relinque et al., 2013). En tal sentido, para este estudio se seleccionaron a 10 participantes de universidades públicas y privadas de Lima. De los 10 participantes, siete (07) están cursando actualmente una carrera universitaria, mientras que tres (03) son egresados quienes cursaron el último ciclo de la carrera durante el primer semestre del año 2020. El criterio de inclusión fue que los participantes cursaran al menos un semestre académico completo bajo la modalidad educativa virtual adoptada durante la pandemia y participaran voluntariamente en el estudio.

Tabla 1. Características de los participantes que colaboraron con el presente estudio.

\begin{tabular}{lccc}
\hline Participante & Sexo & Edad & Actual condición educativa \\
\hline Participante 1 & Hombre & 47 & Recién Egresado \\
Participante 2 & Mujer & 27 & Recién Egresado \\
Participante 3 & Mujer & 16 & Estudiante \\
Participante 4 & Mujer & 17 & Estudiante \\
Participante 5 & Mujer & 27 & Estudiante \\
Participante 6 & Mujer & 16 & Estudiante \\
Participante 7 & Hombre & 17 & Estudiante \\
Participante 8 & Mujer & 18 & Estudiante \\
Participante 9 & Hombre & 26 & Recién Egresado \\
Participante 10 & Hombre & 27 & Estudiante \\
\hline
\end{tabular}

\subsection{Técnicas de Recolección de Datos}

Con base a las recomendaciones de Jansen (2013), en la presente investigación se utilizó la encuesta virtual como técnica de recopilación de información para fines cualitativos. La encuesta fue elaborada en el programa de administración de encuestas Google Forms. El formulario de dicha encuesta, estuvo conformado por 07 (siete) de preguntas de carácter abierto, los cuales se muestran a continuación.

Tabla 2. Preguntas del formulario aplicado a los participantes de la investigación.

\begin{tabular}{|c|c|c|}
\hline \multirow{2}{*}{$\begin{array}{l}\text { 1. ¿Te afectó la repentina transición de la modalidad } \\
\text { educativa presencial a modalidad educativa virtual } \\
\text { durante la pandemia? }\end{array}$} & Sí & \multirow{2}{*}{ Realice un comentario breve de su respuesta } \\
\hline & No & \\
\hline \multirow{2}{*}{$\begin{array}{l}\text { 2. ¿Consideras que el material educativo } \\
\text { proporcionado por tus docentes fue útil para tu } \\
\text { formación académica? }\end{array}$} & Sí & \multirow{2}{*}{ Realice un comentario breve de su respuesta } \\
\hline & No & \\
\hline \multirow{2}{*}{$\begin{array}{l}\text { 3. ¿Consideras que tus docentes tenían las } \\
\text { competencias adecuadas en el manejo de las } \\
\text { Tecnologías de la Información y Comunicación (TIC)? }\end{array}$} & Sí & \multirow{2}{*}{ Realice un comentario breve de su respuesta } \\
\hline & No & \\
\hline \multirow{2}{*}{$\begin{array}{l}\text { 4. ¿Las plataformas virtuales puestas a disposición } \\
\text { por tu centro de estudios permitieron llevar a cabo las } \\
\text { clases virtuales sin inconvenientes? }\end{array}$} & Sí & \multirow{2}{*}{ Realice un comentario breve de su respuesta } \\
\hline & No & \\
\hline \multirow{2}{*}{$\begin{array}{l}\text { 5. Según tu experiencia, ¿Consideras que se le debe } \\
\text { dar mayor importancia a las clases virtuales como una } \\
\text { modalidad complementaria de las clases } \\
\text { presenciales? }\end{array}$} & Sí & \multirow[b]{2}{*}{ Realice un comentario breve de su respuesta } \\
\hline & No & \\
\hline \multirow{2}{*}{$\begin{array}{l}\text { 6. En tu caso, ¿Crees que la modalidad educativa } \\
\text { virtual es (fue) útil para tu desarrollo profesional? }\end{array}$} & Sí & \multirow{2}{*}{ Realice un comentario breve de su respuesta } \\
\hline & No & \\
\hline $\begin{array}{l}\text { 7. Por último, en una frase breve ¿Cómo definirías, o } \\
\text { qué sentimientos te genera (generaba) la nueva } \\
\text { modalidad de educación virtual? }\end{array}$ & - & - \\
\hline
\end{tabular}




\subsection{Procedimiento}

Se contactó vía telefónica a 16 candidatos (entre estudiantes universitarios y recién egresados) para explicarles el propósito de la investigación, responder las dudas surgidas y asegurarles la confidencialidad de los datos proporcionados. Del total de candidatos con quienes se sostuvo una conversación informativa, 10 participantes mostraron disponibilidad de tiempo y habían cursado como mínimo un ciclo académico vía online. Seguidamente a los postulantes que estaban dispuestos a colaborar con la investigación les fue remitida la encuesta, con la instrucción de desarrollar en extenso con un mínimo de 50 palabras un comentario sobre la respuesta. Posteriormente fueron contactados vía telefónica para expresar los agradecimientos correspondientes. Es pertinente mencionar que, el informe final del presente estudio será facilitado a todas las personas que colaboraron con esta evaluación.

\subsection{Análisis de Datos}

Después de haber reunido la información proporcionada por los 10 participantes, se procedió con el análisis de los datos cualitativos, siguiendo los procedimientos descritos por Urbano (2016), el cual está constituido por los siguientes pasos: 1. Obtención de la información, 2. Ordenación de la información, 3. Codificación de la información y por último 4. Integración de la información. Con respecto al primer paso, las respuestas proporcionadas por los participantes en el formulario de Google Forms se descargaron en formato Libro de Excel (.xlsx), luego las respuestas textuales se ordenaron en un procesador de textos (Microsoft Word) para su mayor entendimiento y para proceder con la codificación de la información. La codificación de la información se realizó mediante la asignación de etiquetas y categorías a las respuestas proporcionadas por los participantes, en función de: 1. Palabras y expresiones recurrentes en las respuestas y/o sus sinónimos. 2. Atributos. Por último, se unieron las categorías obtenidas en la etapa de codificación integrando los contenidos.

\section{Resultados y Discusiones}

En base a las respuestas obtenidas por los estudiantes universitarios, se muestran los siguientes resultados:

\section{- Pregunta 1}

El $60 \%$ de los participantes afirmaron que la repentina transición de la modalidad de enseñanza presencial a la nueva modalidad virtual al inicio de la pandemia, si les había afectado. De las respuestas textuales que brindaron se resaltaron las palabras "perjudicado", "fastidiado" e "incómodo". Por ejemplo, la respuesta de uno de los participantes fue:

"Se me hace difícil entender los temas ya que en presencial podemos apreciar al profesor desarrollar los ejercicios o temas sobre las demás áreas" (Participante 4).

El fragmento anterior demuestra que, en aquellas asignaturas de naturaleza práctica o aplicativa, la presencia del docente es de suma importancia para guiar a los estudiantes en el proceso de aprendizaje. Evidentemente, tal y como lo menciona (Garzozi-Pincay, Solórzano-Méndez \& Sáenz-Ozaetta, 2020), en la modalidad virtual la interacción entre los alumnos y docentes se ve limitada, pues al prevalecer una interacción indirecta y en muchos casos interrumpida por diversos factores, la resolución de dudas o el intercambio de ideas es limitado. 
El resto de participantes sostenían que la nueva modalidad no les había afectado, y que se habían adaptado rápidamente. Un claro ejemplo de dicha afirmación es:

"No tuve problemas en la transición [...], incluso tuve algunos profesores que seguían haciendo clase en pizarra como en las clases presenciales" (Participante 10).

De esta respuesta se puede desprender que, a pesar de que la metodología de enseñanza experimentó un cambio drástico en tan poco tiempo, en los centros de estudios se siguieron impartiendo todos los cursos establecidos en el currículo para la formación de las carreras universitarias. Esto último, parece ser la idea adecuada, sin embargo, para aquellas asignaturas en las que las cuestiones aplicativas y prácticas predominan por sobre las cuestiones teóricas podría representar una desventaja para estudiantes (Cóndor, 2020; Villafuerte, Bello, Pantaleón \& Bermello, 2020).

\section{- Pregunta 2}

Todos los participantes afirmaron que los materiales educativos proporcionado por sus docentes sí fueron útiles para su formación académica. Lo que indica que los docentes, independientemente de sus competencias en el manejo de las Tecnologías de la Información y Comunicación (TIC), si prepararon materiales adecuados para sus alumnos. Un ejemplo es lo que mencionó el participante 10 en la pregunta 1.

Seguidamente, al solicitarles a los participantes que describan las cualidades de dichos materiales educativos, se codificaron atributos como "claro y conciso", "apropiado para estudiar", y "material de calidad". A continuación, se muestran algunas respuestas de los estudiantes que validan dichos adjetivos:

"El profesor es muy claro en sus conceptos y nos ayuda mucho a entender todo" (Participante 1).

"...Pero lo más determinante creo que fue las clases grabadas, ya que si te quedaba alguna duda podías volver a ver las clases en cualquier momento" (Participante 10).

"Los videos si fueron útiles porque así entendía más el tema" (Participante 3).

Es importante resaltar la detección de que, una de las afirmaciones que más valoraban los estudiantes con respecto a los recursos educativos, fue la importancia de tener acceso a las clases grabadas. Todos los participantes que mencionaron esta facilidad, aludieron que era un recurso que les permitía repasar las lecciones y era favorable para fomentar el aprendizaje autónomo. Lo que es cónsono con la investigación de Zainuddin \& Halili, (2016).

\section{- Pregunta 3}

El 90\% de encuestados consideró que los docentes manejaban adecuadamente las TICs, puesto que las clases que les impartían eran didácticas. Así también, dichas clases eran presentadas adecuadamente mediante diferentes recursos multimedia. Los estudiantes percibieron el apoyo de los docentes durante el uso de los TICs, tal y como indica la participante 2:

"no he tenido problemas con los profesores ya que nos envían videos claros de acuerdo al tema del curso".

Lo que es apoyado por la participante 5 :

"los profesores manejaban correctamente la plataforma institucional y promovían la participación grupal".

Sin embargo, el participante 10 expresó que: "al comienzo les costó mucho a los profesores adaptarse a algunas plataformas como webex, zoom o meet [...], pero a medida que avanzaba el curso los docentes se adaptaron". 
Es comprensible la falta de manejo de las TICs desde el inicio de la pandemia, puesto que ha significado cambios en el método tradicional de aprendizaje. Para Vélez (2020), ello ha sido un gran reto para los docentes, debido a que tienen que asegurar la misma calidad educativa con la inclusión de las TICs en el proceso educativo. La incorporación de las TICs ha sido gradual, pero esencial para facilitar el aprendizaje y apoyar didácticamente con el uso nuevas herramientas multimedia (Areth, Castro-Martínez \& Rodríguez, 2015). Ante este contexto, es necesario promover un mayor uso y actualización permanente en herramientas multimedia con el fin de que, los estudiantes cuenten con las competencias necesarias para aprender y los docentes, de enseñar desde la virutalidad.

\section{- Pregunta 4}

El $90 \%$ de los participantes mostraron conformidad con las plataformas virtuales puestas a su disposición para su aprendizaje, tal y como indica el participante 10:

"en mi caso mi casa de estudios uso la plataforma webex para las clases y tenía un campus virtual en el que se subían distintos materiales y esa plataforma facilitaba todo..."

De igual manera, se observa que se sienten apoyados por su institución:

"la plataforma era fácil de manejar y además, había un curso para aprender a manejarla" (participante 5).

Es esencial que las universidades brinden un constante apoyo en el desarrollo de competencias multimedia de los estudiantes. Esta consideración es subrayada por Casillas \& Ramírez (2016), quienes indican que el uso de plataformas virtuales y otros medios de comunicación, inducen a que los docentes preparen material didáctico y actualizado, dejando de lado viejas prácticas como las fotocopias, especialmente, en universidades. En esta misma perspectiva para Meseguer, Hernández \& Ramírez (2016), estas plataformas flexibilizan el aprendizaje y promueven la autonomía y compromiso del estudiante.

No obstante, la participante 4 mostró una respuesta negativa ante el prolongado uso de plataformas virtuales para la visualización de sus cursos:

"Tuve dificultad ya que presentaba problemas sobre el Internet, la señal y no podía estar en clase".

Ello evidencia que, no necesariamente el problema radica en el uso de la plataforma virtual, pero que al presentar problemas de conexión acentúa el abrupto cambio de aprendizaje por medios virtuales.

\section{- Pregunta 5}

El $60 \%$ de participantes muestra una mayor inclinación de dar mayor importancia a las clases bajo la modalidad virtual. Esto debido a que, según lo expresado, les permite manejar mejor sus tiempos y una mayor flexibilidad en sus horarios de clases, destacando algunas respuestas:

"Las clases virtuales nos sorprendieron bastante ...ya que todos no estábamos preparados para esta modalidad... pero en el transcurso del tiempo hemos experimentado nuevos métodos que nos permite desarrollarnos más" (participante 2).

A pesar de que, varios estudiantes muestran esta respuesta positiva no es en su totalidad, puesto que algunos aún mantienen una alta preferencia por las clases presenciales.

"Pese que todo marchó muy bien de forma virtual, prefiero las clases presenciales" (participante 1).

"No porque en clases virtuales hay personas que no entienden muy bien por eso se retiran de los estudios" (participante 6). 
Lo mencionado por el participante 6 , constituye una amenaza y una oportunidad para promocionar la alfabetización multimedia, así como el desarrollo de competencias computacionales requeridas para el posterior ejercicio profesional. Asimismo, es evidente la demanda de actualización de las plataformas y mallas curriculares con el fin de que, se logre ofrecer una alta calidad digital educativa (Cueva, 2020).

\section{- Pregunta 6}

El $60 \%$ de los participantes consideraron que la modalidad educativa virtual fue útil para su formación profesional y aprendizaje de cursos teóricos. Sus respuestas estaban relacionadas con las expresiones "necesario circunstancialmente", "beneficioso y novedoso", "útil" y "optimización del tiempo". Las siguientes afirmaciones son ejemplos de lo mencionado:

"Hubo cursos que eran teóricos...y en el ciclo virtual cambiaron la modalidad, ahora se hacían simulaciones, exposiciones o informes y creo que en esta modalidad se aprendía mejor algunos conceptos" (Participante 10).

“...me ayudo a controlar mejor mis tiempos y ser eficiente en la presentación de mis trabajos con un mayor grado de comodidad" (Participante 5).

Las respuestas precedentes favorecen la consolidación del modelo educativo virtual. No obstante, el resto de participantes consideraban lo contrario, indicando expresiones como "Desfavorable y disparejo", "Inútil e inentendible" y "Demasiado teórico" respecto a las clases virtuales. Por ejemplo, algunas de las respuestas proporcionadas fueron:

"No me fue muy útil ya que no podía hacer prácticas y todo lo necesario que se solía hacer de manera presencial" (Participante 7).

"Para algunos estudiantes no fue útil, porque tenían muchos problemas con la conectividad a internet" (Participante 2).

De estas respuestas, se infiere que existen asignaturas académicas que confrontan limitaciones, por cuanto demandan la presencia física de docentes y estudiantes, como es el caso de los laboratorios. Asimismo, la brecha en el acceso al servicio de internet constituye uno de los factores más sensibles que afecta la percepción de los estudiantes motivando el rechazo las clases virtuales.

\section{- Pregunta 7}

Los participantes respondieron en la gran mayoría positivamente resaltando "simplicidad" y "autonomía". Por ejemplo, las afirmaciones de tres participantes fueron:

"Bueno tenemos que adaptarnos, ya que el virus nos ha enseñado nuevas modalidades de convivencia" (Participante 2).

"Casi en familia con nuevos compañeros y nuevos profesores, felicidad" (Participante 8).

"Muy buena, ya era hora de entrar en la era digital" (Participante 9).

Todas estas respuestas son alentadoras, debido a que, reflejan el compromiso que tienen los estudiantes para hacer frente a adversidades externas como la emergencia sanitaria mundial. Sin embargo, también algunos participantes tenían una apreciación negativa destacando sentimientos de "inconformidad", "ansiedad", "tristeza" y "resignación". Siendo claros ejemplos de ello, las siguientes afirmaciones:

"Con la modalidad virtual no aprendo igual que con la modalidad presencial" (Participante 3).

"Me genera desesperación ya que para mí no es un buen método para aprender, más bien se nos dificulta" (Participante 4).

Las dos afirmaciones expuestas, si bien, no representan a la mayoría de los participantes, sugieren que hay una gran cantidad de estudiantes con problemas similares. 
Por lo tanto, en caso de seguir prolongado el uso de las clases virtuales hasta que se controle el estado de emergencia sanitaria mundial, es necesario enfatizar el seguimiento, orientación y apoyo a los estudiantes que presentan problemas cognitivos e instrumentales para acceder a una educación de calidad.

\section{Conclusiones}

La repentina implementación de la modalidad educativa virtual, debido al contexto sanitario mundial del Covid 19, indujo hacia la alfabetización digital forzosa de estudiantes y docentes en todos los niveles educativos. En ese orden, el objetivo de ésta investigación fue describir el nivel de satisfacción respecto al nuevo sistema de educación virtual por estudiantes universitarios en Lima, en época de pandemia Covid-19. Los resultados indican que, para los estudiantes universitarios participantes en el estudio, la adaptación se produjo progresivamente, desde inicios categorizados como "difíciles hacia la adaptación" al nuevo esquema virtual, al que aún perciben deficiente en relación a la modalidad presencial. Aunado a ello, se evidenció que los problemas de conectividad generan asimetrías en acceso a la educación.

La evaluación del desempeño docente en general fue favorable destacando la sincresis entre los modos tradicionales de enseñanza y la modalidad virtual bajo esquemas de aula invertida, donde se evidencia las modificaciones en el orden de la taxonomía de Blohm. No obstante, persisten dudas en relación a las potencialidades de la educación virtual frente a las demandas de los cursos donde el desempeño presencial es ineludible como las prácticas de laboratorio, lo que constituye una brecha aun por estudiar.

Metodológicamente este estudio se propuso explorar una estrategia de abordaje de investigación fenomenológica, fundamentado en herramientas virtuales como propuesta de investigación cualitativa en el contexto de la pandemia. La investigación indica que los estudios fenomenológicos encuentran amplios ámbitos de desarrollo mediante la implementación de herramientas virtuales, superando las limitaciones logísticas para estudios en ámbitos de difícil acceso como en los casos de situación de conflicto armado ubicuidad, violencia de género, segregación entre otros.

\section{Agradecimentos}

A los participantes del presente estudio por brindarnos su tiempo y apoyo.

\section{Referencias}

Areth, J., Castro-Martínez, J., \& Rodríguez, H. (2015). La educación virtual en Colombia: exposición de modelos de deserción. Apertura, 7 (1), 1-10.

Cáceres-Muñoz, J., Jiménez, A., \& Martín-Sánchez, M. (2020). Cierre de Escuelas y Desigualdad Socioeducativa en Tiempos del Covid-19. Una Investigación Exploratoria. Revista Internacional de Educación para la Justicia Social, 9, 199-221.

Cáceres-Piñaloza, K. F. (2020). Educación virtual: Creando espacios afectivos, de convivencia y aprendizaje en tiempos de COVID-19. CienciAmérica, 9(2), 38.

Cóndor, O. (2020). Educar en tiempos de COVID-19. CienciAmérica, 9(2), 31-37.

Cueva-Gaibor, D.A.. (2020). La tecnología educativa en tiempos de crisis. Conrado, 16(74), 341-348.

De La Fuente, H., Marzo, M., \& Reyes, M. J. (2010). Analysis of satisfaction of students of the faculty of engineering of universidad de Talca. Revista Chilena de Ingeniería, 18(3), 350-363.

Durán, R., Estay-Niculcar, C., \& Álvarez, H. (2015). Adoption of good virtual education practices in higher education. Aula Abierta, 43(2), 77-86. 
Garzozi-Pincay, R., Solórzano-Méndez, V., \& Sáenz-Ozaetta, C. (2020). Ventajas y Desventajas de la relación enseñanza-aprendizaje en la educación virtual. Tecnología Educativa Revista CONAIC, 3(3), 58-62.

González, L. (2019). El Aula Virtual como Herramienta para aumentar el Grado de Satisfacción en el Aprendizaje de las Matemáticas. Información Tecnológica, 30(1), 203-214. http://dx.doi.org/10.4067/S0718-07642019000100203

Hernández, R., Fernández, C., \& Baptista, M. del P. (2014). Metodología de la Investigación (6ª ed.) México, D.F.:McGraw Hill Education.

Hernández-Silva, A. \& Tecpan Flores, S. (2017). Aula invertida mediada por el uso de plataformas virtuales: un estudio de caso en la formación de profesores de física. Estudios Pedagógicos XLIII, (3), 193-204.

Jansen, H. (2013). La lógica de la investigación por encuesta cualitativa y su posición en el campo de los métodos de investigación social. Paradigmas, 5(1), 39-72.

Manterola, C., Quiroz, G., Salazar, P., \& García, N. (2019). Metodología de los tipos y diseños de estudio más frecuentemente utilizados en investigación clínica. Revista Médica Clínica Las Condes, 30(1), 36-49.

Martínez-Garcés, J., \& Garcés-Fuenmayor, J. (2020). Competencias digitales docentes y el reto de la educación virtual derivado de la covid-19. Educación y Humanismo, 22(39), 1-16.

Meseguer, A., Hernández, A., \& Ramírez, A. (2016). Curso de Son Jarocho en modalidad virtual. In A. Ramírez \& M. A. Casillas (Eds.), Háblame de TIC: Educación Virtual y Recursos. 31-49.

Olivera Carhuaz, E. (2020). Satisfacción académica de los estudiantes universitarios en el marco de la educación virtual. Revista Científica de Comunicación Social Bausate, (2), 16-24.

Ramírez, A., \& Casillas, M. A. (2016). Una metodología para la incorporación de las TIC al currículum universitario. In A. Ramírez \& M. A. Casillas (Eds.), Háblame de TIC: Educación Virtual y Recursos. 31-49.

Relinque, C. S., Moral, G. Del, \& González, M. T. (2013). Consejos prácticos para escribir un artículo cualitativo publicable en Psicología. Psychosocial Intervention, 22(1), 71-79.

Salgado, A. N. (2007). Investigación cualitativa: diseños, evaluación del rigor metodológico y retos. Liberabit, 13(13), 71-78.

Sierra, C. A. (2013). La educación virtual como favorecedora del aprendizaje autónomo. Panorama, 5(9), 75-87.

Trujillo, M., Marulanda, C., \& Bustamante, D. (2009). La educación virtual, análisis y gestión en las universidades de Manizales. Revista Virtual Universidad Católica Del Norte, (28), 1-23.

Urbano, P. A. (2016). Análisis de datos cualitativos. Revista Fedumar Pedagogía y Educación, 3(1), $113-126$.

Vélez, R. M. (2020). Retos de las universidades latinoamericanas en la educación virtual. Revista Virtual Universidad Católica del Norte, (59), 1-3.

Villafuerte, J. S., Bello, J. E., Pantaleón, Y., \& Bermello, J. O. (2020). Rol de los docentes ante la crisis del Covid-19, una mirada desde el enfoque humano. Revista Electrónica Formación y Calidad Educativa (REFCalE), 8(1), 134-150.

Zainuddin, Z. \& Halili, H. S (2016). Flipped classroom research and trends from different fields of study. International Review of Research in Open and Distributed Learning, 17(3), 313-340. 